OPINION PAPER

\title{
JUMP HEIGHT IS A POOR INDICATOR OF LOWER LIMB MAXIMAL POWER OUTPUT: THEORETICAL DEMONSTRATION, EXPERIMENTAL EVIDENCE AND PRACTICAL SOLUTIONS
}

Jean-Benoit Morin ${ }^{1,3}$, Pedro Jiménez-Reyes², Matt Brughelli³ ${ }^{3}$ Pierre Samozino ${ }^{4}$

1 Université Côte d'Azur, LAMHESS, Nice, France

${ }^{2}$ Faculty of Physical Sciences and Sport, Catholic University of San Antonio, Murcia, Spain

${ }^{3}$ Sports Performance Research Institute New Zealand, Auckland University of Technology, Auckland, New Zealand

${ }^{4}$ Univ Savoie Mont Blanc, Laboratoire Interuniversitaire de Biologie de la Motricité, EA 7424, F73000 Chambéry, France

Corresponding author

Pr Jean-Benoit Morin

Faculty of Sport Science

261 Boulevard du Mercantour

06205 NICE, France

Tel: 0033489153956

Email: jean-benoit.morin@unice.fr

Web: jbmorin.net

Twitter: @jb_morin

DOI: 10.31236/osf.io/6nxyu

Cite as:

Morin, J., Jiménez-Reyes, P., Brughelli, M., \& Samozino, P. (2018, September 4). Jump height is a poor indicator of lower limb maximal power output: theoretical demonstration, experimental evidence and practical solutions. https://doi.org/10.31236/osf.io/6nxyu 


\title{
KEY POINTS
}

1. Despite a widespread use, we contended that jump height as measured during vertical jump tests is not a good indicator of lower limb power or maximal power output capability.

2. We showed this based on several confounding factors: body mass, push-off distance, individual force-velocity profile and optimal force-velocity profile. Some experimental data were also shown and discussed to further illustrate the not very-good correlation between jump height and power output.

3. Finally, in order to address this issue, we discussed the possible practical solutions, and advocate for the use of a simple, accurate computation method based on jump height as an input.

\begin{abstract}
Lower limb maximal power output $\left(P_{\max }\right)$ is a key physical component of performance in many sports. During squat jump (SJ) and countermovement jump (CMJ) tests, athletes produce high amounts of mechanical work over a short duration to displace their body mass (i.e. the dimension of mechanical power). Thus, jump height has been frequently used by the sports science and medicine communities as an indicator of $P_{\max }$. However, in this article, we contended that SJ and CMJ height are in fact poor indicators of $P_{\max }$ in trained populations.

To support our opinion, we first detailed why, theoretically, jump height and $P_{\max }$ are not fully related. Specifically, we demonstrated that individual body mass, distance of push-off, optimal loading and force-velocity characteristics confound the jump height- $P_{\max }$ relationship.

We also discussed the poor relationship between SJ or CMJ height and $P_{\max }$ measured with a force plate based on data reported in the literature, which added to our own experimental evidence. Finally, we discussed the limitations of existing practical solutions (regression-based estimation equations and allometric scaling), and advocated using a valid, reliable and simple field-based procedure to compute individual $P_{\max }$ directly from jump height, body mass and push-off distance. The latter may allow researchers and practitioners to reduce bias in their assessment of $P_{\max }$ by using jump height as an input with a simple yet accurate computation method, and not as the first/only variable of interest.
\end{abstract}




\section{INTRODUCTION}

Vertical jump tests such as the squat jump (SJ) and countermovement jump (CMJ) have been long and widely used to assess an athlete's lower limb ballistic performance and indirectly quantify their mechanical power output capability, i.e. the ability to produce mechanical work over the duration of the push-off [1-3]. Although measuring technologies have differed over the past century, vertical jump tests are still very popular due to their simple and easy implementation, good reproducibility and time-energy saving feature [4]. The main metric used to describe jumping performance is maximal height after the athlete leaves the ground. For these reasons, vertical jump tests have been popularized with classic testing batteries and procedures $[1-3,5,6]$. Since maximal power output $\left(P_{\max }\right)$ is a key physical component of performance in many sports, and because vertical jumps like SJ and CMJ require athletes to produce mechanical (potential) work over a short duration to displace their body mass (i.e. the dimension of mechanical power), jump height is frequently used as an indicator of $P_{\max }$ [3]. For example, in a recent survey about strength and conditioning practice in elite rugby union [4], the authors report that the most commonly used test of muscular power was maximum CMJ height.

In this article, we contend that maximum SJ and CMJ height are in fact poor indicators of lower limb $P_{\max }$ in trained populations. To support our opinion, we have first detailed the theoretical reasons explaining why jump height and $P_{\max }$ are not fully related. We then provided experimental evidence of the poor to very poor associations between SJ or CMJ height and $P_{\text {max. }}$. Finally, we detailed a simple and field-based procedure to accurately compute $P_{\max }$ from SJ or CMJ height measurements. The latter may allow researchers and practitioners to improve their assessment of $P_{\max }$, using SJ or CMJ height as inputs with a simple yet accurate computation method [7-9], of which calculation spreadsheets are freely available online

(https://www.researchgate.net/publication/320146284_JUMP_FVP_profile_spreadsheet).

\section{THEORETICAL DEMONSTRATION}

We detailed the three main factors that account for the discrepancy between jump height and lower limb $P_{\max }$ in this section: individual push-off distance $\left(h_{\mathrm{PO}}\right)$, optimal loading $\left(L_{\mathrm{opt}}\right)$ and force-velocity (Fv) profile.

\subsection{Push-off distance}

The SJ and CMJ motions require the athlete to produce (mainly with their lower limb extensor muscles) and exert force onto the supporting ground during the push-off phase. This phase lasts from the starting (SJ) or most downward (CMJ) position classically set at $\sim 90^{\circ}$ knee angle to the take-off position with fully extended lower limb. The distance over which the lower limb extends during the push-off phase $\left(h_{\mathrm{PO}}\right)$ thus represents the distance over which muscles will generate force and mechanical work [8]. Thus, it is clear that two athletes with different lower limb segment lengths (even for a given same body stature) may have very different $h_{\text {Po }}$ values while pushing from the $90^{\circ}$ starting position to their extended limb take-off position. In turn, should they have the same maximal SJ or CMJ height, they would present different push-off distances and times, leading to very different levels of power output. The athlete with a greater $h_{\text {Po }}$ will also show, ceteris paribus, a lower mechanical power output, and vice versa. The theoretical importance of $h_{\text {PO }}$ in the SJ height performance equation has been demonstrated by Samozino et al. [10] and is not negligible. Furthermore, Markovic et al. [11] showed that body mass and CMJ depth (which is equivalent to $h_{\mathrm{PO}}$ ) clearly confounded the relationship between muscle power output and jumping performance. These authors also suggested that the effect of $h_{\text {PO }}$ should be controlled when interpreting the inter-individual results of SJ or CMJ tests. 
This crucial importance of $h_{\text {PO }}$ helps to explain the outstanding jumping capability in some animals with muscular force outputs that are not clearly superior to human athletes [12]. In the sports performance context, not taking into account the effect of $h_{\text {PO }}$ on SJ or CMJ performance may result in misleading interpretations of jump tests as predictors of power output, with a bias toward underestimating the power capability of an athlete with a small $h_{\text {PO }}$ and conversely overestimating that of an athlete with a great $h_{\mathrm{PO}}$. This issue may be of particular importance in young age categories (in which variable $h_{\text {PO }}$ may be observed among athletes of the same chronological age) when using SJ or CMJ height as a screening-selection test. Finally, $h_{\mathrm{PO}}$ is not only determined by individual anthropometrical factors, but also, when it is freely chosen, by the self-selected starting position (SJ) or countermovement depth (CMJ). The latter are associated to muscle force-length properties, training history or sport specificities, which supports the fact that $h_{\mathrm{PO}}$ should be carefully controlled.

\subsection{Optimal loading}

In any type of exercise allowing maximal muscular exertions (e.g. throwing, jumping, cycling, running), the loading conditions influence movement velocity (following the laws of motion), which in turn influences power output, with $P_{\max }$ achieved only in an optimal loading condition ( $L_{\text {opt }}$ [13-15]. For example, should the external loading be too heavy or too light, the conditions would lead the athlete to produce higher levels of force or velocity, respectively, but in both cases this would be associated with sub-maximal levels of power output. This is well described by the second degree polynomial relationship observed between movement velocity output and power output in various types of exercises [16-20].

In the specific context of vertical jumps, external loading influences the power output $[14,15,21]$ and $P_{\max }$ is only produced in $L_{\text {opt }}$ conditions. Thus, $P_{\max }$ is achieved by a given individual in a SJ or CMJ only if their individual $L_{\text {opt }}$ is equal to their body mass. This corresponds to the "maximum dynamic output hypothesis" [13,22-24]. However, due to individual neuromuscular characteristics, and training history, some athletes have a $L_{\mathrm{opt}}$ that differs from their own body mass, and would need either positive (additional load) or negative (assistance, unloaded conditions) loading to jump with their $L_{\mathrm{opt}}[23,25]$.

As a conclusion to this section, it is important to distinguish the power output developed during a maximal (i.e. all-out) single effort (e.g. SJ or CMJ) to the maximal power output. Inferring that SJ or CMJ height is associated with optimal loading conditions (and thus $P_{\max }$ ) is only correct if the individual athlete tested has their own body mass as $L_{\mathrm{opt}}$, which is a substantial assumption.

\section{3. $\quad$ Force-velocity profile and optimal profile}

Samozino et al. [9,21] have clearly shown, using a theoretical approach [10] confirmed by experimental evidence $[19,26]$, that SJ height was almost entirely explained by the following variables: $P_{\max }, h_{\mathrm{PO}}$ and the slope of the Fv profile. Thus, added to the previous section about the influence of $h_{\mathrm{PO}}$, it is clear that the relationship between maximal jump height and $P_{\max }$ is confounded by the balance between the athletes' force and velocity capabilities, as described by their Fv profile. The two implications shown by Samozino et al.'s works are that (i) for a same $h_{\text {PO }}$ and $P_{\text {max }}$, different levels of SJ height may be achieved depending on the Fv profile of the athletes and (ii) the theoretical maximal SJ height for a given individual (given their $h_{\mathrm{PO}}$ and $P_{\max }$ ) is only reached if the actual Fv profile is equal to the calculated optimal Fv profile (and in this case, body mass is the $\left.L_{\mathrm{opt}}\right)[8,10,19,26]$. In other words, an athlete with an optimal Fv profile for jump height will have his own body mass as $L_{\mathrm{opt}}$, and thus produce $P_{\max }$ during vertical jump without additional load.

The relative influences of $h_{\mathrm{PO}}, P_{\mathrm{max}}$ and Fv profile on SJ performance are summarized in the following equation, that has been validated experimentally $[19,26]$ :

$h_{\max }=\frac{h_{\mathrm{PO}}^{2}}{2 g}\left(\sqrt{\frac{S_{F v} o p t^{2}}{4}+\frac{2}{h_{P O}}\left(2 \sqrt{-P_{\max } S_{F v} o p t}-g\right)}+\frac{S_{F v} o p t}{2}\right)^{2}$ 
In this equation, $h_{\max }$ is the maximal possible SJ height reached with an optimal Fv profile $S_{F v} O p t$. Note that these demonstrations have been initially published using SJ as the jump modality, but subsequent works by Jimenez-Reyes et al. have shown that similar conclusions could be drawn for the CMJ modality [7].

A practical summary of the above-mentioned theoretical points is that the relationship between SJ or CMJ height is clearly confounded by individual anthropometrical and physiological factors inherent to each athlete tested. Not taking these factors into account may lead to bias when quantifying $P_{\max }$ via single jump tests without additional load, as will be evidenced with the following simulations (Table 1) and the experimental measurements discussed in section 3.

\section{EXPERIMENTAL EVIDENCE}

To support our point that the relationship between SJ or CMJ height and lower limb $P_{\max }$ is poor to very poor with experimental evidence, we will first list and discuss the published correlations between these variables. Then, we will present some experimental data we have collected.

Table 2 shows a few examples limited to studies reporting jump height measurements and the magnitude of their correlation to lower limb maximal power output as measured with the reference force plate method, and expressed in Watts. Independently from their statistical significance, these correlations are of rather low magnitude to accurately estimate power output and $P_{\max }$ from jump height, on an individual basis.

Table 2. Pearson's correlation coefficients and linear regression coefficients between jump height and lower limb peak instantaneous power measured with force plates and expressed in Watts. The population age is displayed as an average value or range of values for the group. SJ: Squat Jump. CMJ: Counter Movement Jump.

\begin{tabular}{|c|c|c|c|c|}
\hline Reference & $\begin{array}{c}\text { Number and Type of } \\
\text { subjects }\end{array}$ & $\begin{array}{l}\text { Type } \\
\text { of } \\
\text { jump }\end{array}$ & $\begin{array}{c}\text { Correlation } \\
\text { Coefficient and } \\
\text { Magnitude } \\
\end{array}$ & $\begin{array}{c}\text { Linear } \\
\text { Regression } \\
\text { Coefficient }\end{array}$ \\
\hline $\begin{array}{l}\text { Bridgeman } \\
\text { et al. } 2016 \\
{[27]}\end{array}$ & $\begin{array}{l}12 \\
\text { Males }(25.4 \pm 3.5 \mathrm{yrs}) \\
\text { strength trained }\end{array}$ & $\mathrm{CMJ}$ & 0.62 - Large & 0.38 \\
\hline $\begin{array}{l}\text { Young et al. } \\
2011 \text { [28] }\end{array}$ & $\begin{array}{l}23 \\
\text { Males }(22.3 \pm 2.1 \mathrm{yrs}) \\
\text { elite Australian } \\
\text { football players }\end{array}$ & $\mathrm{CMJ}$ & 0.41 - Moderate & 0.17 \\
\hline $\begin{array}{l}\text { Amonette } \\
\text { et al. } 2012 \\
{[29]}\end{array}$ & $\begin{array}{l}415 \\
\text { Males }(15.7 \pm 2.8 \text { yrs }) \\
\text { soccer, American } \\
\text { football, kinesiology } \\
\text { students }\end{array}$ & $\mathrm{CMJ}$ & 0.65 - Large & 0.42 \\
\hline $\begin{array}{l}\text { Markovic } \\
\text { and Jaric. } \\
2007[30]\end{array}$ & $\begin{array}{l}159 \\
\text { Males (18-25 yrs), } \\
\text { physical education } \\
\text { students }\end{array}$ & $\begin{array}{c}\mathrm{SJ} \\
\mathrm{CMJ}\end{array}$ & $\begin{array}{c}0.47 \text { - Moderate } \\
0.66 \text { - Large }\end{array}$ & $\begin{array}{l}0.22 \\
0.44\end{array}$ \\
\hline
\end{tabular}

Although the magnitude of the correlation between jump height and $P_{\max }$ may be improved through allometric scaling (see 4. Practical solutions), the relationship between these two variables is not acceptable, in our opinion, to accurately infer $P_{\max }$ from SJ or CMJ measurements, on an individual athlete's basis. Not to mention the use of this estimation in the context of sports performance monitoring or athlete's screening/selection process. 
By adding experimental data to illustrate our point, we have measured SJ or CMJ height and $P_{\max }$ values in trained populations (sprint, weightlifting and elite rugby league competitors) within various published and in-review research protocols [7,31]. Both jump height and $P_{\max }$ were assessed using a force plate system, and $P_{\max }$ was determined as the apex of the load-power relationship for several loaded jump conditions (see $[19,26]$ for details). Note that most studies cited in this paper did not use this approach to determine $P_{\max }$, but rather the "peak"or "maximal" value of instantaneous power output measured during a single jump.

It is clear from this dataset (Fig. 1 and 2) of trained to elite athletes (n=90 for SJ and $n=68$ for CMJ) that the correlations between $P_{\max }$ and jump height were not systematically very large ( $\mathrm{r}=0.493$ to 0.877 ), although improved (not for $\mathrm{CMJ}$ in sprinters and jumpers, Figure 2) when normalizing $P_{\max }$ to body mass.

a.

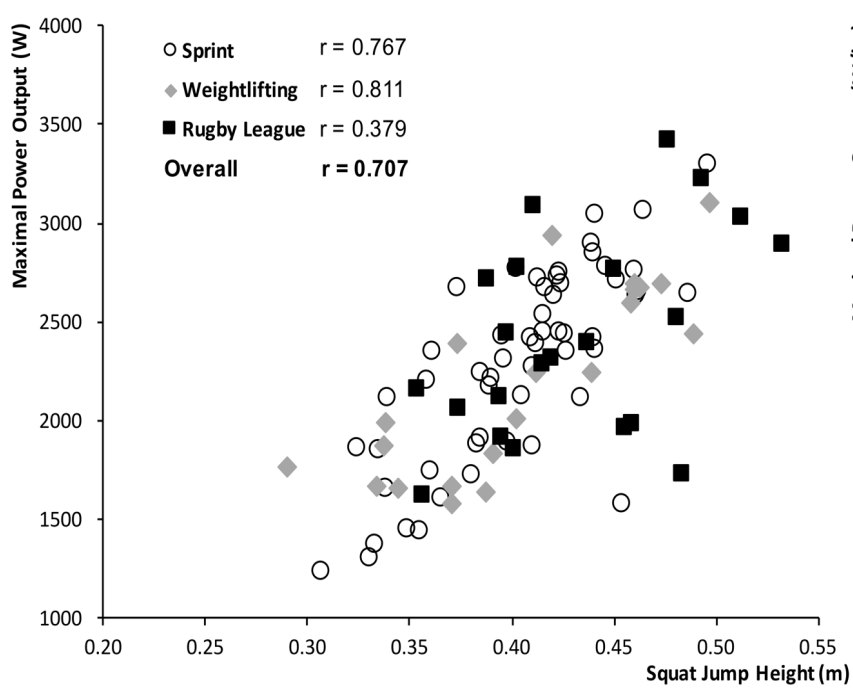

b.

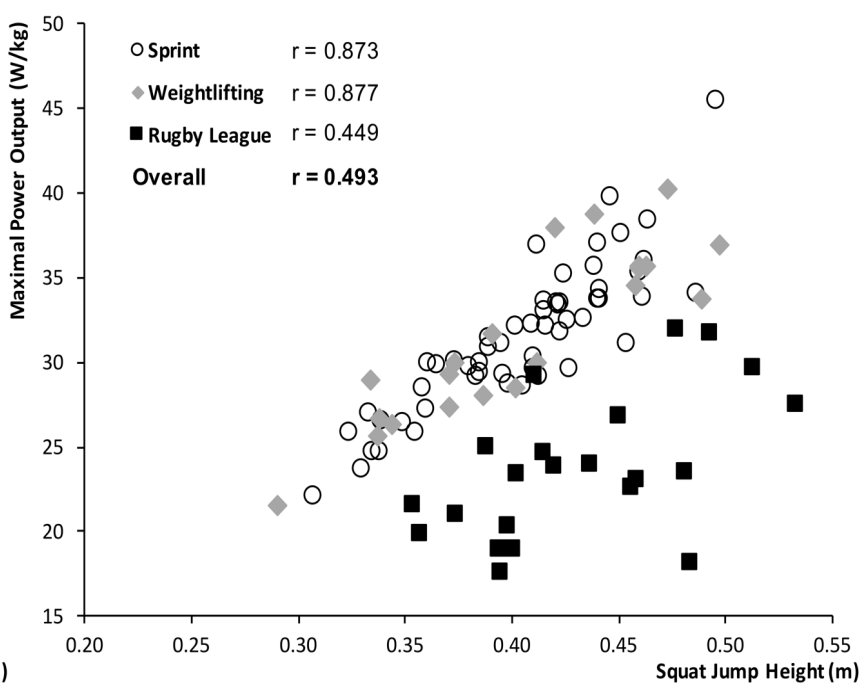

Fig. 1. Correlations (Pearson's r) between squat jump height and absolute (panel a.) or relative to body mass (panel b.) maximal power output in highly-trained sprinters, weightlifters and rugby league players
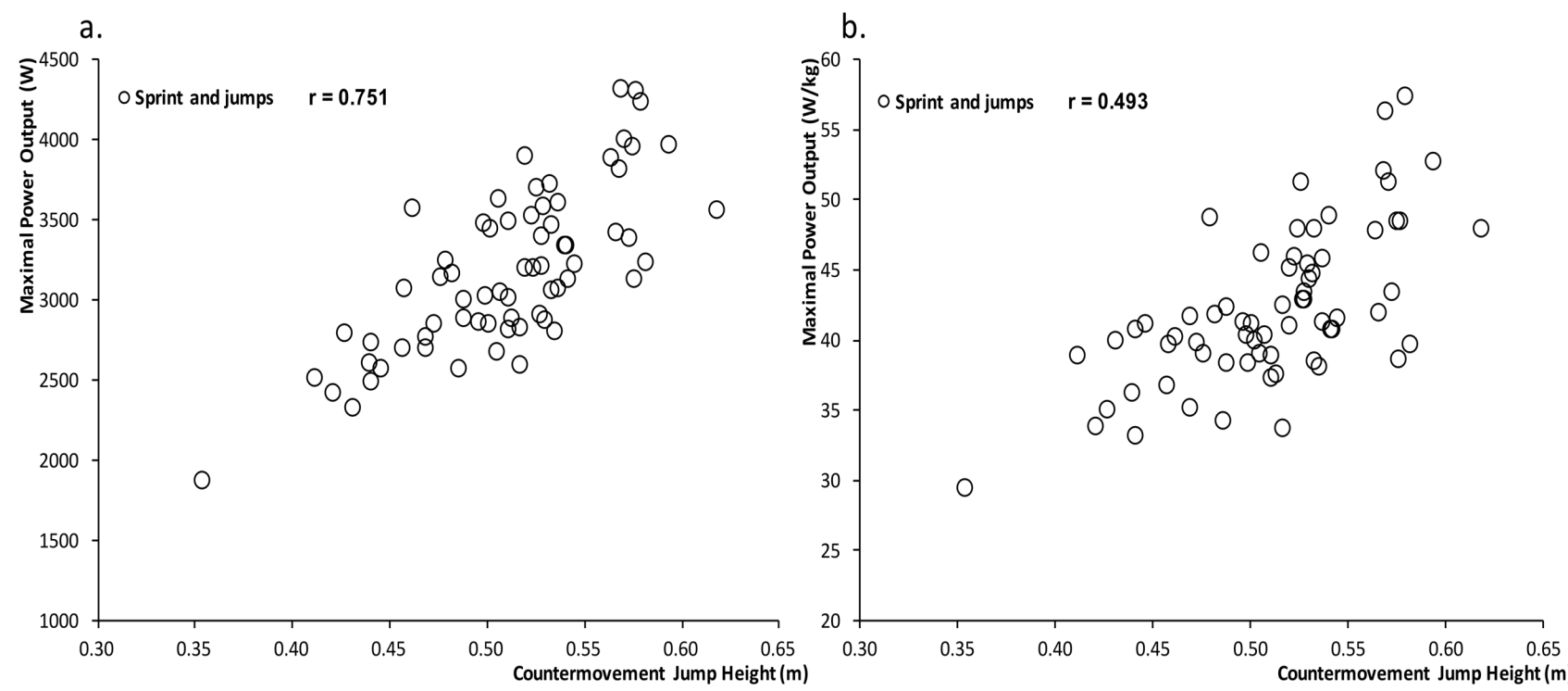

Fig. 2. Correlations (Pearson's r) between countermovement jump height and absolute (panel a.) or relative to body mass (panel b.) maximal power output in highly-trained sprinters and jumpers. 
Furthermore, the corresponding low regression coefficients (overall $\mathrm{r}^{2}<0.56$ ) show that a large part of the variance in $P_{\max }$ is not directly explained by the variance in jump height. Jump height and $P_{\max }$ are significantly correlated, but the magnitude of the correlations is not high enough to estimate the latter accurately from the former. In line with the theoretical points listed above, possible explanations for the relatively low correlations observed for the overall group (Fig. 1) include: variable $h_{\mathrm{PO}}$ values among athletes, heterogeneous individual Fv profiles and optimal profiles, and in turn optimal load that differs from body mass for some athletes (which was the case for most rugby players).

Finally, two recent studies show that athlete's absolute lower limb power output capability could not be appropriately categorized or estimated, in most sports, from jump height measurements $[32,33]$. Among the practical solutions to solve this issue, the current authors propose to use estimations based on regressions, and allometric scaling of raw, absolute power values. In the next section we will discuss these possibilities and their limitations, and support the use of a more direct, accurate and practical method we recently validated.

\section{PRACTICAL SOLUTIONS}

\subsection{Regression-based estimation equations}

One solution to better estimate $P_{\max }$ from SJ or CMJ measurements has been the use of regressionbased equations. Briefly, a regression (usually linear) equation is generated from actual jump height and $P_{\max }$ measured with reference methods (e.g. force plate) in a set of subjects. Then, the accuracy of the $P_{\max }$ values "predicted" by the equation (which inputs are often jump height and a few simple variables such as body mass or height) is assessed by comparison to those obtained with the reference methods. Numerous regression equations have been published to estimate $P_{\max }$ (e.g.[34-39]). However, although practical, this estimation method has several major limitations. The first limitation is the lack of theoretical rationale supporting the link between $P_{\max }$ jump height and body mass, notably via a simple weighted addition of the two latter variables (see 2. Theoretical demonstration). Another limitation of such equations is that they are population-dependent, which may lead to a lower accuracy for estimating power on an individual basis. For instance, Quagliarella et al. [40] performed a detailed comparative study of several equations, and reported very high error values (>50\%) and concluded that the use of $P_{\max }$ estimates, can only be useful to compare the performance of groups of subjects. Discrepancies and equation-dependant accuracy of prediction were also observed in other comparative studies $[8,36,41,42]$, making the use of regression-based estimates irrelevant for an individually accurate determination of lower limb $P_{\max }$.

\subsection{Allometric scaling}

Accounting for the potential influence of body dimensions (mostly stature and mass) on $P_{\max }$ as estimated through SJ or CMJ measurements can be done by scaling the raw, absolute value of $P_{\max }$ to a power of these body dimensions [43,44]. For instance, scaling $P_{\max }$ to body mass (SJ) or body mass and countermovement depth (CMJ) at the power 1 resulted in markedly increased correlation coefficients with jump height [11]. This led to researchers recommending the use of allometric scaling to more accurately estimate $P_{\max }$ from jump height $[30,32,33]$. In particular, Markovic and Jaric [30] proposed that the most justified and accurate scaling was when muscle power was proportional to body mass raised to the power of 0.67 (and even more accurately 0.75 as discussed by these authors) to account for the theory of geometric similarity, that states that some physiological features of mammals bodies (e.g. muscle physiological cross-sectional area) are not directly proportional to body mass [45].

However, allometric scaling is a correction method that is also based on assumptions, and may not apply correctly in heterogeneous populations, and/or in the case of very specific body composition and especially skeletal muscle mass as developed through years of specific training in elite athletes. Kons et al. recently showed that the improvement of correlations between jump 
height and $P_{\max }$ through allometric scaling was highly sport-specific [33]. Interestingly, Fig. 1 shows that the correlation strongly differs between elite rugby players and other athletes compared when normalizing $P_{\max }$ to body mass, leading to an overall lower correlation when all athletes are considered. This tends, again, to show that indirect methods (regression-based estimates or allometric scaling) may not totally solve the individual accuracy issue discussed in this article. To this aim, an alternative method has been proposed 10 years ago [8], that directly computes jumping power output and $P_{\max }$, based on a biomechanical model and simple inputs, as described in the following section.

\subsection{Direct computation using a simple field method}

In order to solve most of the previously described issues and provide a simple and practical yet accurate way to compute force, velocity and power output during a SJ push-off, Samozino et al. have proposed and validated a computation method in 2008 [8]. This method, based on macroscopic modelling of body mass displacement and the associated mechanical external work, requires only body mass, $h_{\mathrm{PO}}$ and jump height. The initial reliability and concurrent validity was tested against reference force plate measurements [8], and was confirmed in subsequent studies from other authors, including additional load conditions and application to CMJ [7,46,47].

In this method, the mean power produced during a jump was computed as:

$P=m g\left(\frac{h}{h_{P O}}+1\right) \sqrt{\frac{g h}{2}}$

Eq. 2

with $m$ the body mass, $g$ the gravitational acceleration, $h_{\text {PO }}$ the vertical push-off distance, and $h$ the jump height. In this work, jump height was defined as the aerial distance covered by the center of mass between the take-off and vertical apex instants.

It has also been confirmed that $P_{\max }$ can be accurately computed from multiple-load jump protocols as:

$P_{\text {max }}=\frac{\left(F_{0} V_{0}\right)}{4}$

Eq. 3

$F_{0}$ and $V_{0}$ being the force and velocity-axes intercepts of the linear Fv relationship obtained from the multiple-load measurements [19]. However, since many human subjects (especially those with no extensive force- or velocity-oriented training) show a $P_{\max }$ that is close to their SJ power output, $P_{\max }$ might be correctly computed by using the SJ power value.

Like all biomechanical models, this method has assumptions and limitations that have been discussed extensively elsewhere $[8,21,48]$. In addition, these computations lead to mean values during the push-off phase, as opposed to peak values estimated in many studies (e.g. [27,49]). That said, even if instantaneous and mean values may reasonably be assumed to be linked during vertical jumps, mean values (i.e. averaged over the whole movement) appeared to be more representative of the muscular effort [9,50].

That being said, given the reliability and external validity of this equation to compute SJ and CMJ power output (and also easy to obtain $P_{\max }$ ), we believe these limitations do not induce meaningful errors and bias, and are largely outweighed by the possibility to simply and practically calculate jump power, from jump height. This is all the more important since jump height measurements/estimations alone show some clear limitations, as discussed in the previous sections. Interestingly, this method has been integrated in a very practical and reliable [51-53] smartphone app (MyJump2), allowing cheaper yet accurate computations of jump height and power output, from a simple high-speed video recording of the jumps. 


\section{CONCLUSIONS}

Given (i) the several limitations of using jump height measurements to accurately infer an athlete's lower limb power output capabilities, and (ii) the possibility to more accurately calculate mean power output during SJ or CMJ based on body mass, lower limb length and jump height using Equation 2, we strongly recommend that sports performance, medicine, and research colleagues use the method proposed by Samozino et al. [8,26], instead of the standard measure of jump height alone. This may lead to a richer level of information, from the very same input measurements.

\section{ACKNOWLEDGEMENTS}

The authors wish to thank Dr Slobodan Jaric, who has influenced some of the concepts and thinking behind this work, through his major contributions to the field, and his friendly discussions and remarks. We were very sad to learn that Dr Jaric had passed away during the writing process of this paper.

Authors have no source of funding or conflict of interest to declare regarding this paper.

\section{REFERENCES}

1. Cronin J, Sleivert G. Challenges in understanding the influence of maximal power training on improving athletic performance. Sports Med. 2005;35:213-34.

2. McMaster DT, Gill N, Cronin J, McGuigan M. A Brief Review of Strength and Ballistic Assessment Methodologies in Sport. Sports Med. 2014;44:603-23. Available from: http://www.ncbi.nlm.nih.gov/pubmed/24497158

3. Vandewalle H, Pérès G, Monod H. Standard anaerobic exercise tests. Sports Med. 1987;4:26889.

4. Jones TW, Smith A, Macnaughton LS, French DN. Strength and Conditioning and Concurrent Training Practices in Elite Rugby Union. J Strength Cond Res. 2016;30:3354-66.

5. Sargent DA. The Physical Test of a Man. Am Phys Educ Rev. 1921;26:188-94

6. Bosco C, Komi PV. Potentiation of the mechanical behavior of the human skeletal muscle through prestretching. Acta Physiol Scand. 1979;106:467-72.

7. Jiménez-Reyes P, Samozino P, Pareja-Blanco F, Conceição F, Cuadrado-Peñafiel V, GonzálezBadillo JJ, et al. Validity of a Simple Method for Measuring Force-Velocity-Power Profile in Countermovement Jump. Int J Sports Physiol Perform. 2017;12:36-43.

8. Samozino P, Morin JB, Hintzy F, Belli A. A simple method for measuring force, velocity and power output during squat jump. J Biomech. 2008;41:2940-5.

9. Samozino P. A Simple Method for Measuring Lower Limb Force, Velocity and Power Capabilities During Jumping. In: Morin JB, Samozino P, editors. Biomechanics of Training and Testing. Springer International Publishing; 2018. p. 65-96.

10. Samozino P, Morin JB, Hintzy F, Belli A. Jumping ability: A theoretical integrative approach. J Theor Biol. 2010;264:11-8.

11. Markovic S, Mirkov DM, Nedeljkovic A, Jaric S. Body size and countermovement depth confound relationship between muscle power output and jumping performance. Hum Mov Sci. 2014;33:203-10.

12. Channon AJ, Usherwood JR, Crompton RH, Gunther MM, Vereecke EE. The extraordinary athletic performance of leaping gibbons. Biol Lett. 2012;8:46-9.

13. Jaric S, Markovic G. Leg muscles design: the maximum dynamic output hypothesis. Med Sci Sports Exerc. 2009;41:780-7.

14. Markovic G, Jaric S. Positive and negative loading and mechanical output in maximum vertical jumping. Med Sci Sports Exerc. 2007;39:1757-64. 
15. Soriano MA, Jiménez-Reyes P, Rhea MR, Marín PJ. The Optimal Load for Maximal Power Production During Lower-Body Resistance Exercises: A Meta-Analysis. Sports Med. 2015;45:1191-205.

16. Cross MR, Brughelli M, Samozino P, Morin JB. Methods of Power-Force-Velocity Profiling During Sprint Running: A Narrative Review. Sports Med. 2017;47:1255-69.

17. Dorel S, Couturier A, Lacour J-R, Vandewalle H, Hautier C, Hug F. Force-Velocity Relationship in Cycling Revisited. Med Sci Sport Exerc. 2010;42:1174-83.

18. Rabita G, Dorel S, Slawinski J, Sàez-de-Villarreal E, Couturier A, Samozino P, et al. Sprint mechanics in world-class athletes: a new insight into the limits of human locomotion. Scand J Med Sci Sports. 2015;25:583-94.

19. Samozino P, Rejc E, Di Prampero PE, Belli A, Morin JB. Optimal force-velocity profile in ballistic movements-Altius: Citius or Fortius? Med Sci Sports Exerc. 2012;44:313-22.

20. Yamauchi J, Ishii N. Relations between force-velocity characteristics of the knee-hip extension movement and vertical jump performance. J Strength Cond Res. 2007;21:703-9.

21. Samozino P. Optimal Force-Velocity Profile in Ballistic Push-off: Measurement and Relationship with Performance. In: Morin JB, Samozino P, editors. Biomechanics of Training and Testing. Springer International Publishing; 2018. p. 97-119.

22. Suzovic D, Markovic G, Pasic M, Jaric S. Optimum Load in Various Vertical Jumps Support the Maximum Dynamic Output Hypothesis. Int J Sports Med. 2013;34:1007-14.

23. Pazin N, Berjan B, Nedeljkovic A, Markovic G, Jaric S. Power output in vertical jumps: does optimum loading depend on activity profiles? Eur J Appl Physiol. 2013;113:577-89.

24. Jaric S, Markovic G. Body mass maximizes power output in human jumping: a strengthindependent optimum loading behavior. Eur J Appl Physiol. 2013;113:2913-23.

25. Loturco I, Nakamura FY, Tricoli V, Kobal R, Cal Abad CC, Kitamura K, et al. Determining the Optimum Power Load in Jump Squat Using the Mean Propulsive Velocity. PLoS One. 2015;10:e0140102.

26. Samozino P, Edouard P, Sangnier S, Brughelli M, Gimenez P, Morin JB. Force-velocity profile: Imbalance determination and effect on lower limb ballistic performance. Int J Sports Med. 2014;35:505-10.

27. Bridgeman LA, McGuigan MR, Gill ND, Dulson DK. Relationships Between Concentric and Eccentric Strength and Countermovement Jump Performance in Resistance Trained Men. J Strength Cond. Res. 2018;32:255-60.

28. Young W, Cormack S, Crichton M. Which jump variables should be used to assess explosive leg muscle function? Int J Sports Physiol Perform. 2011;6:51-7.

29. Amonette WE, Brown LE, De Witt JK, Dupler TL, Tran TT, Tufano JJ, et al. Peak vertical jump power estimations in youths and young adults. J Strength Cond Res. 2012;26:1749-55.

30. Markovic G, Jaric S. Is vertical jump height a body size-independent measure of muscle power? J Sports Sci. 2007;25:1355-63.

31. Jiménez-Reyes P, Samozino P, Brughelli M, Morin JB. Effectiveness of an individualized training based on force-velocity profiling during jumping. Front Physiol. 2017;7:677.

32. Ache-Dias J, Dal Pupo J, Gheller RG, Külkamp W, Moro ARP. Power Output Prediction From Jump Height and Body Mass Does Not Appropriately Categorize or Rank Athletes. J Strength Cond Res. 2016;30:818-24.

33. Kons RL, Ache-Dias J, Detanico D, Barth J, Dal Pupo J. Is Vertical Jump Height an Indicator of Athletes' Power Output in Different Sport Modalities? J Strength Cond Res. 2018;32:70815.

34. Canavan PK, Vescovi JD. Evaluation of power prediction equations: peak vertical jumping power in women. Med Sci Sports Exerc. 2004;36:1589-93.

35. Johnson D, Bahamonde R. Power output estimates in university athletes. J Strength Cond Res. 1996;10:161-6. 
36. Lara-Sánchez AJ, Zagalaz ML, Berdejo-Del-Fresno D, Martínez-López EJ. Jump peak power assessment through power prediction equations in different samples. J Strength Cond Res. 2011;25:1957-62.

37. Lara A, Alegre L, Abian J, Jimenez L, Urena A, Aguado X. The selection of a method for estimating power output from jump performance. J Hum Mov Stud. 2006;50:399-410.

38. Sayers SP, Harackiewicz D V, Harman EA, Frykman PN, Rosenstein MT. Cross-validation of three jump power equations. Med Sci Sports Exerc. 1999;31:572-7.

39. Shetty AB. Estimation of leg power: a two-variable model. Sport Biomech. 2002;1:147-55.

40. Quagliarella L, Sasanelli N, Belgiovine G, Moretti L, Moretti B. Power Output Estimation in Vertical Jump Performed by Young Male Soccer Players. J Strength Cond Res. 2011;25:1638-46.

41. Hertogh C, Hue 0. Jump evaluation of elite volleyball players using two methods: jump power equations and force platform. J Sports Med Phys Fitness. 2002;42:300-3.

42. Tessier J-F, Basset F-A, Simoneau M, Teasdale N. Lower-Limb Power cannot be Estimated Accurately from Vertical Jump Tests. J Hum Kinet. 2013;38:5-13.

43. Jaric S. Muscle strength testing: use of normalisation for body size. Sports Med. 2002;32:615-31.

44. Markovic G, Jaric S. Movement performance and body size: the relationship for different groups of tests. Eur J Appl Physiol. 2004;92:139-49.

45. Alexander RM. Principles of animal locomotion. Princeton University Press. 2002.

46. Giroux C, Rabita G, Chollet D, Guilhem G. What is the best method for assessing lower limb force-velocity relationship? Int J Sports Med. 2015;36:143-9.

47. Palmieri G, Callegari M, Fioretti S. Analytical and multibody modeling for the power analysis of standing jumps. Comput Methods Biomech Biomed Engin. 2015;18:1564-73.

48. Hatze H. Validity and reliability of methods for testing vertical jumping performance. J Appl Biomech. 1998;14:127-40.

49. Barker LA, Harry JR, Mercer JA. Relationships between countermovement jump ground reaction forces and jump height, reactive strength index, and jump time. J Strength Cond Res. 2018;32:248-54.

50. Andrews JG. Biomechanical measures of muscular effort. Med Sci Sports Exerc. 1983;15:199-207.

51. Balsalobre-Fernández C, Glaister M, Lockey RA. The validity and reliability of an iPhone app for measuring vertical jump performance. J Sports Sci. 2015;33:1574-9.

52. Stanton R, Kean CO, Scanlan AT. My Jump for vertical jump assessment. Br J Sports Med. 2015;49:1157-8.

53. Stanton R, Wintour S-A, Kean CO. Validity and intra-rater reliability of MyJump app on iPhone 6s in jump performance. J Sci Med Sport. 2017;20:518-23. 